\title{
Representing numbers: prime and irrational
}

\author{
RINA ZAZKIS \\ Faculty of Education, Simon Fraser University, Vancouver, \\ British Columbia, Canada, V5A 1S6
}

(Received 20 July 2004)

\begin{abstract}
This article draws an analogy between prime and irrational numbers with respect to how these numbers are defined and how they are perceived by learners. Excerpts are presented from two research studies: a study on understanding prime numbers by pre-service elementary school teachers and a study on understanding irrational numbers by pre-service secondary school teachers. Considering the results of these studies, the author calls for further attention in teaching to transparent features in representation of numbers and suggests several strategies on how this may be achieved.
\end{abstract}

\section{What do irrational numbers and prime numbers have in common?}

The readers are invited to examine for a minute their answer or reaction to this question, before continuing reading.

When we asked students this question, the most common response was either a puzzled look, like saying without saying 'What kind of stupid question is that?' or simply 'nothing', accompanied by a shrug. The 'nothing' referred to the empty intersection of the two sets. When we asked colleagues, the most common response was a puzzled look, followed by something like 'Oh! Never thought of this!'

In this paper we draw an unusual analogy between prime numbers and irrational numbers. This analogy refers to the lack of 'transparent' representation (the term is explained below) for these numbers. We discuss how this may influence students' perceptions of numbers and present an obstacle for understanding.

\section{Definitions and images}

Roughly speaking, irrational numbers are those that cannot be represented as a quotient and prime numbers are those that cannot be represented as a product. Inserting mathematical rigour into this rough observation we obviously acknowledge that the above-mentioned quotient refers to $a / b$, where $a$ is an integer and $b$ is a non-zero integer, whereas the above-mentioned product is a product

Email: rina_zazkis@sfu.ca 
of natural numbers greater than 1. However, our focus in what follows is not on the rigorous constraints but on the words 'cannot be represented' - that is, identifying concepts not by what they are but by what they are not and focusing on representation.

Of course the above description is a vague part of students' 'concept image' à la Tall and Vinner [1] rather than a definition. Tall and Vinner use the term concept image to describe 'the total cognitive structure that is associated with the concept, which includes all the mental pictures and associate properties and processes' (p. 152). As such, 'cannot be represented' is a part of how these concepts are perceived. A mathematical definition of an irrational number relies on the concept of real number, which is formally defined using Dedekind cuts or Cauchy sequences. However, long before these ideas are accessible for students, the existence of certain representations is a distinguishing feature. That is, a rational number is a number that can be represented as $a / b$, where $a$ is an integer and $b$ is a nonzero integer, and an irrational number is a number that cannot be represented as a ratio of integers. An equivalent definition for an irrational number refers to infinite nonrepeating decimal representation - that is, the non-existence of a finite or repeating representation.

Further, a formal mathematical definition for a prime is that a prime number is a natural number that has exactly two factors. (We prefer this definition to the one that refers to divisibility by only 1 and 'itself' as it helps exclude the number 1 from the list of primes). However, in a recent study on pre-service teachers' understanding of prime numbers [2] we noticed that neither definition has sufficient explanatory power for students. Consider, for example, replies of several pre-service teachers when asked to describe a prime number:

Sally: Prime numbers cannot be divided by anything other than 1 and itself.

Tom: Primes are those that cannot be factored, yeah, like cannot be factored any further.

Karen: Prime number would be a number that would be divisible only by 1 and itself, it means it wouldn't have any other factor, besides these two factors, 1 and itself are the only factors.

Jenny: Prime numbers have only two factors. They are not having other factors to be broken down into.

We notice that the formal definition of primes, that was presented to students in the textbook and by the instructor, is either not mentioned at all in these descriptions of primes or is accompanied by 'negative' descriptions. Participants appear to think of prime numbers not in terms of properties of these numbers but in terms of properties these numbers do not hold; prime numbers 'cannot be divided', 'cannot be factored' or 'wouldn't have/are not having any other factor'. Zazkis and Liljedahl [2] reported that negative descriptions appeared in the responses of 15 out of 18 participants either in their initial description of prime numbers (e.g. Sally and Tom) or in their additional explanations (e.g. Karen and Jenny).

In summary, negative description is what irrationals and primes have in common, either as a working definition or as a common perception. 


\section{Representations: transparent and opaque}

Lesh et al. [3] introduced the distinction between 'transparent' and 'opaque' representations. According to these researchers, a transparent representation has no more and no less meaning than the represented idea(s) or structure(s). An opaque representation emphasizes some aspects of the ideas or structures and de-emphasizes others. Borrowing the terminology from Lesh et al. [3] in drawing the distinction between transparent and opaque representations, Zazkis and Gadowsky [4] focused on representations of numbers introducing the notion of relative transparency and opaqueness. Namely, they suggested that all representations of numbers are opaque in the sense that they always hide some of the features of a number, although they might reveal others, with respect to which they would be transparent. For example, representing the number 784 as $28^{2}$ emphasizes - makes transparent - that it is a perfect square, but de-emphasizes - leaves opaque - that it is divisible by 98 . Representing the same number as $13 \times 60+4$ makes it transparent that the remainder of 784 on dividing by 13 is 4 , but leaves opaque its property of being a perfect square. In general, we say that a representation is transparent with respect to a certain property, if the property can be 'seen' or derived by considering the given representation.

Zazkis and Liljedahl [2] extended further the notions of transparency of representation of specific numbers to the representation of sets of numbers possessing the same property through the use of algebraic notation. That is, for a whole number $k, 17 k$ is a transparent representation for a multiple of 17 , as this property is embedded or 'can be seen' in this form of the representation. However, it is impossible to determine whether $17 k$ is a multiple of 3 by considering the representation alone. In this case we say that the representation is opaque with respect to divisibility by 3 .

Applying the notions of opaqueness and transparency we suggest that an infinite non-repeating decimal representation (such as $0.010011000111 \ldots$ ) is a transparent representation of an irrational number (that is, irrationality can be derived from this representation), while representation as a common fraction is a transparent representation of a rational number (that is, rationality is embedded in the representation).

However, there is no 'finite' transparent representation for an irrational number (the above example involves an infinite process). Consider, for example, the classical proof of the irrationality of $\sqrt{2}$. It starts with assuming, by contradiction, that the number is rational. This assumption is essential because a rational number can be represented and therefore can be manipulated, unlike its irrational counterpart.

Likewise, we usually denote a prime number by $p$, but this representation is opaque in every sense. Primality cannot be derived from this representation in a way that, for example, oddness of a number can be derived from the representation $2 k+1$. Representing a number as a product of two natural numbers greater than 1 is a transparent representation for a composite number. However, there is no transparent representation for a prime number.

We believe that lack of transparent representation in both cases contributes to the 'negative' perception of both sets, that is, perception of numbers in terms of properties they do not possess. In what follows we present excerpts from two research studies that examined understanding of primes and irrational 
numbers, respectively. Both studies focused on the role representations (or lack thereof) play in students' understanding of numbers.

\section{On primes and representations}

In our recent research study on understanding primality [2] the following was one of the questions presented to a group for 116 pre-service elementary school teachers.

Consider $F=151 \times 157$. Is $F$ a prime number?

Circle YES/NO and explain your decision.

The participants in this study were students enrolled in a 'Principles of mathematics for teachers' course, which is a core course for certification at the elementary level. The question was presented to participants upon completion of the unit on elementary number theory.

Of the 74 students who claimed correctly that $F$ is composite only 52 justified this by its structure as a product, focusing the explanation on the definition of either prime or composite numbers. A popular response was to calculate $F$ and apply the learned algorithms of checking for primality. This resulted in both correct and incorrect conclusions, as many students checked divisibility of $F$ only by a few 'small' primes. For example, Kathy described her strategy in the following way:

$151 \times 157=23707, \sqrt{23707}=154$

Check all prime numbers lower than 154 to see if the number is prime and if none of them can divide 23707 then the number is prime.

However, this general description was followed by the list of prime numbers up to 23 and a conclusion that $F$ was prime. Kathy's strategy is not incorrect, but rather unnecessary and inelegant in the given case; it is the incomplete implementation of the strategy that led to a wrong conclusion.

Other popular responses included variations on the following:

- Both 151 and 157 are prime numbers, and 2 prime numbers multiplied together are going to give another prime number

- It is prime because the last digit in the number is 7 and the sum of the digits is the number 19. 19 is a prime number and is not divisible by anything but itself and 1 . So $F$ is prime.

It is impossible, given a relatively large number, to conclude its primality without a careful 'checking' by, for example, applying the familiar algorithm. However, when a number is presented as a product, it is composite by definition, it has factors. The unifying feature in incorrect responses or inappropriate justifications for correct decisions is lack of attention to this transparent feature - the product of whole numbers - in the representation of number $F$.

\section{On irrationals and representations}

In our recent research study on understanding irrationality [5] the following was one of the questions presented to a group of 46 pre-service secondary mathematics teachers. 
Consider 53/83. Let's call this number $M$. In performing this division, the calculator display shows 0.63855421687 . Is $M$ a rational or an irrational number? Explain.

(Note that the numbers are carefully chosen so that the repeating nature of the decimal representation is 'opaque' on a calculator display. The length of the period in this case is 41 digits.)

The participants in this study had at least two calculus courses in their background, and the majority held either majors or minors in mathematics. However, only about $60 \%$ of participants provided a correct response that was followed by an appropriate justification - that is, $M$ is rational since it is a quotient of two whole numbers. We accept the criticism that this question was intentionally misleading, but it was our goal to check what kind of distraction could be caused by a calculator.

Other responses included the following:

- $53 / 83$ is irrational because there is no pattern in the decimal 0.63855421687 .

- 53/83 is rational because it terminates (calculator shows 0.63855421687 ).

- 53/83 could be rational or irrational - I cannot tell whether digits will repeat because too few digits are shown. They might repeat or they might not.

- There is no way of telling if 53/83 is rational - unless you actually do the division which could take you forever. Digits might terminate at a millionth place or they might start repeating after a millionth place.

- It is possible that a number is rational and irrational at the same time. For example, there are fractions that have non-repeating non-terminating decimals, yet they can be represented as $a / b$.

- It is easy to turn a fraction into a decimal. But there is no easy, general way of turning a decimal into a fraction. Looking at a decimal, unless it is a terminating decimal, you cannot tell if it is rational or not.

In these responses we identify several overlapping themes: application of an incorrect or incomplete definition, distraction by a calculator display, and lack of awareness of the relationship between fractions and repeating decimals. However, the overall tendency in these responses is lack of attention to or ignorance of the representational feature - the quotient of integers - that is transparent.

\section{The role of representations in mathematics education}

The issue of representation is not new to mathematics education. However, it has recently attracted fresh attention and examination in mathematics education research and practice $[6,7]$. The discussion of representations in mathematics is often related to qualitatively different representational systems, such as written symbols, pictures or manipulative models. However, our focus here is on representations that unravel properties of numbers. What role do these representations play in mathematics and in learning mathematics?

\subsection{Tools for manipulation and communication}

Consider for example the sum of two odd numbers. How do we prove that this sum is even? One popular way is to take two odd numbers to be $2 k+1$ and 
$2 n+1$ and to calculate their sum to be $(2 k+1)+(2 n+1)=2 k+2 n+2=$ $2(k+n+1)$. Note that we started with a representation and interpreted the conclusion based on another representation. As such, we have shown that in this case representation serves as a tool for manipulating objects and also as a tool for communicating properties of objects. An alternative visual or pictorial proof also relies on representing objects, manipulating the representation and interpreting the results of manipulation.

In a similar fashion, consider the sum of two rational numbers. It is always rational because the representation

$$
\frac{a}{b}+\frac{c}{d}
$$

can be manipulated to be a quotient

$$
\frac{a d+c b}{b d}
$$

(considering for rigour that $b d \neq 0$ because $b \neq 0$ and $d \neq 0$ as well as the closure in addition and multiplication of whole numbers).

Having a representation in hand allows an individual to detach him/ herself from the meaning of this representation and operate on the symbols alone, making the manipulations automatic, and returning later to interpreting the result of the symbolic manipulation [8]. Further, the nature of the manipulation to be performed may influence the choice of representation. It is indisputable that multiplication of large numbers is better manipulated when these numbers are represented in the Hindu-Arabic, rather than the Roman, numeration system. Likewise, for the purpose of multiplying complex numbers, the polar representation is preferable to the ordered pair representation of complex numbers.

Consider now the sum of two irrational numbers. Sirotic and Zazkis [9] reported that 19 out of $46(41 \%)$ pre-service secondary school teachers participating in her study claimed that this sum (in fact the sum of two positive irrational numbers) is always irrational. Having intuitive disposition towards closure - that is, a belief that an operation with two objects of a certain kind will result in a third object of the same kind [2] - these students did not seek counterexamples, but attempted to justify their intuitive belief with examples or erroneous claims. Two representative examples of such claims are:

- Two numbers that have an infinite number of non-repeating digits to the right of the decimal will have an infinite number of non-repeating digits in their sum.

- You cannot add $\sqrt{2}+\pi$, but you can add their decimal representations; the sum cannot be a terminating decimal.

Sirotic and Zazkis [9] interpreted these results as over-reliance on decimal representation of irrational numbers. It was further noted that due to lack of algorithmic experience students do not perceive expressions such as $1+\sqrt{2}$ as numbers, a perception that would automatically lead to a variety of counter examples. Further we believe that the lack of a finite symbolic representation for irrational number impedes students' ability to manipulate these 
numbers in a way that rational numbers can be manipulated relying on standard representation.

\subsection{Tools for understanding}

Researchers draw strong connections between the representations students use and their understanding $[10,11]$. Understanding is connected to the ability to apply various representations and to choose one appropriate to the problem situation. Janvier [12] describes understanding as a 'cumulative process mainly based upon the capacity of dealing with an 'ever-enriching' set of representations' (p. 67).

Furthermore, representations are considered as a means to form conceptual understanding. The ability to move smoothly between various representations of the same concept is seen as an indication of conceptual understanding and also as a goal for instruction [3]. Likewise, Even [13] suggests that knowledge of different representations is intertwined with knowledge of underlying notions and contexts.

In our recent research on understanding irrational numbers we concluded that students relied on decimal representations rather than common fraction representations. We further observed that the connection between two ways to describe irrational numbers - as lacking representation as a ratio and having infinite non-repeating representation - were not well understood. Zazkis and Sirotic [5] described this lack of connection as a 'missing link' in understanding. Yet again we believe that one of the reasons for this missing link is a lack of transparent symbolic representation for irrational numbers. That is to say, drawing correspondence between decimal and common fractions, finite or repeating decimals correspond to fractions, but infinite non-repeating decimals correspond to objects that lack representation and therefore are hard to grasp.

\subsection{Tools for mental constructions}

Representations are also described in the literature as tools for generalization and abstraction, as expressing generality can be achieved by an appropriate choice of representation. Moreover, according to Kaput [14], possessing an abstract mathematical concept 'is better regarded as a notationally rich web of representations and applications' (p. 61).

Treating mathematical concepts as objects supports the construction of corresponding mental objects in students' mind $[15,16]$. One possible way to treat concepts as objects is to involve them as inputs in mathematical processes, that is, to act on them - to perform operations on them. In order to act on representatives of certain sets of numbers, representation is an asset. We have mentioned above that in order to consider the sum of two odd numbers we represent them as $2 k+1$ and $2 n+1$ and perform the operation of addition by acting on this representation. Of course one can use symbols such as $x$ and $y$ for the two odd numbers, but in such a case no conclusion about the parity of the sum can be drawn from considering $x+y$. Thus, the choice of representation is of importance.

Researchers agree that achieving an object conception of mathematical concepts is challenging. We suggest that the lack of transparent representation for prime numbers as well as for irrational numbers creates an obstacle for acting on them, and, as such, creates an additional difficulty for constructing a mental object. 


\section{Role of examples}

Examples play an important role in learning mathematics. In this section we will argue that this role is even more crucial in cases of irrational and prime numbers where the exemplified objects lack transparent representation.

It is hard to imagine learning mathematics without the consideration of specific examples. Examples provide specific cases that fit the requirements of the definition under discussion. Examples are used to verify statements and to illustrate algorithms or procedures. Examples are said to be important component of expert knowledge [17].

Mason and Pimm [18] claim that when a teacher presents an example, he or she sees its generality and relates to what the example represents. However, a novice learner may notice only particular features of a specific example, paying attention to the example itself and not to what it stands for. Therefore, Mason and Pimm advocate the use of so-called 'generic' examples, examples that represent the general case and attempt to ignore the specifics of the example itself. A similar view is expressed by Lakoff [19] who believes that prototypes can serve the purposes of best and salient examples.

Examples also serve as handy tools when students are required to deal with objects that have not yet been fully constructed in their minds. Considering special cases, or specializing, is a useful heuristic [20,21], the purpose of which is to guide students towards general solutions. However, students who are unable to deal with the general case often base their reasoning on consideration of specific examples. This strategy was described by Hazzan [22] as one of the ways to reduce the level of abstraction.

\subsection{Examples of primes}

Let us turn now to specific examples of prime numbers. As an exercise, please list any ten prime numbers. It is likely that your examples did not include three or four digit numbers. It is likely that only 'small primes', such as 7, 5, 19 or 23 appeared on the list. Those are 'generic' examples of primes. In order to provide an example of a 'large' prime we need to consult prime number tables or choose a number that could be prime (considering the immediately recognizable properties such as odd, not divisible by 5 or 3 ) and check. We cannot easily construct a prime number (the existing computational algorithms are not 'transparent') in the same way that we can construct a number that, for example, leaves a remainder of 7 on division by 17 .

The existence of a transparent representation for a specific number property can help in abstracting and generalizing this property. However, a lack of a transparent representation for prime numbers may lead students to generalize from examples. Of course, generalizing from examples takes place regardless of the available representations; however, with the lack of a transparent representation, this approach may be preferable for constructing an understanding of primes. Based on generalizing from examples and experience, some students believe that prime numbers are small and that every composite number must be divisible by a small prime [23, 2]. A specific illustration of the latter belief is presented in a previous section, in Kathy's solution to determine primality of the number $F$. (She concluded that the number was prime by checking its divisibility by primes up to 23 .) 


\subsection{Examples of irrationals}

Let us turn now to examples of irrational numbers. As an exercise, ask your students to list any 10 irrational numbers. In our experience, some students get stuck after listing $\sqrt{2}$ and $\pi$. Sirotic [24] reports on the following conversation witnessed while observing a mathematics lesson in grade 9:

Student: Is $\pi$ the only irrational number?

Teacher: No, there is also $\sqrt{2}$

Mathematically speaking, the teacher is right. To show that some object is not unique in its kind it is enough to point to another object of the same kind. However, we see in this teacher's response a missed opportunity to open students' minds at least to a variety of examples, if not to an uncountable infinity of irrational numbers. Indeed, $\sqrt{2}$ and $\pi$ are the generic examples for irrational numbers. They also exemplify irrational numbers of different kinds: one is algebraic and one is transcendental. However, the danger in the case of irrational numbers is that what is intended as 'generic' examples may become limiting examples, if other examples are not mentioned.

\subsection{Enriching the repertoire}

If exposure to only a limited set of examples is troublesome, then enriching students' repertoire of examples could be beneficial. Young learners who are exposed only to triangles that are isosceles and presented always as 'standing on the base' have trouble recognizing scalene triangles or even isosceles triangles in other orientations as being 'triangles'. Having recognized this limitation, the authors of current mathematics textbooks for elementary school are careful in presenting different kinds of triangles and varying their spatial orientation. Analogous attempts can be made with numbers, both prime and irrational. Calculator availability should encourage exercises of factoring not only 'nice' numbers like 3600 (the have only $2 \mathrm{~s}, 3 \mathrm{~s}$ and $5 \mathrm{~s}$ in prime decomposition), but also numbers like 31487 $\left(=23 \times 37^{2}\right)$ or $65783(=157 \times 419)$. This experience will ultimately extend the repertoire of examples available to students and provide some experience with larger primes.

And why is it always the 'classical' square root of two? It could be the case that a teacher looking at $\sqrt{2}$ recognizes the generality of this irrational number as a square root of any prime. Zazkis [25] has shown difficulties that undergraduate students experienced in trying to mimic the proof of irrationality of $\sqrt{2}$ to irrationality of $\sqrt{3}$, having trouble in generalizing 'evenness' to divisibility by 3 , or by any prime for that matter. Maybe, if once in a while we mention $\sqrt{17}$, the idea of irrationality will be more easily generalized to include 'square root of any prime'.

As mentioned above, examples are an important component of expert's content knowledge. Examples are usually given or shown to students by a teacher. We suggest that these 'shown' examples, or examples that emerge in solving 'given' problems could be enriched significantly. Further, there is another method of enriching the repertoire of examples available to learners - these are learnergenerated examples, discussed in detail by Watson and Mason [26]. Watson and Mason make a case for a teaching strategy that involves learners in 
generating examples according to given constraints. For example, rather than, or in addition to, engaging in a 'standard' task of calculating the Least Common Multiple (LCM) of two given numbers, learners could be invited to give an example of a pair of numbers that have LCM of, say, 360. When one pair is given, the teacher may ask for another, and another, another... The ultimate goal is for learners to consider the structure of the numbers in all the possible pairs.

Implementing this strategy we can ask students to give an example of an irrational number in a given interval, say between 0 and 1 . Avoiding infinite decimal representation, what is the first example that comes to your mind? Is it the 'generic' $\sqrt{2} / 2$ ? Or maybe $\pi / 6$ ? Further, we can ask students to give an example of a prime number between 400 and 420 . Of course it is impossible to 'pick' such a number without further checking, but it is important for students to 'see' that 419 is a better candidate than 415 or 417 . What knowledge and understanding is needed or can be developed in engaging in a task like this? We believe that by sieving out examples that do not satisfy the requested properties a further understanding of structure can be developed.

\section{Summary and discussion}

The two studies mentioned in this article - understanding primality and understanding irrationality - started independently of each other, but converged to the common theme, the theme of representation. In summary, the common element in both studies is that the questions presented to participants included a transparent representation for a number that is not prime and for a number that is not irrational. The common aspect in participants' responses was that the transparent features of the given representations - that make both questions trivial - were either not recognized or not attended to.

Students' tendencies in addressing the above question can be explained from several theoretical perspectives. The most popular could rely on object/process distinction/duality $[15,16]$ and suggest that some students do not recognize numbers represented as either products or quotients as objects and consider those as processes or instructions to perform operations. Using terminology introduced here, we claimed that these participants did not capitalize upon transparent features of representations. Having explained what makes a representation 'transparent' we acknowledged difficulties in representing prime and irrational numbers. We further claimed that lack of transparent representation increases the role of examples that students are exposed to in their construction of related concepts. We expressed a belief that enriching the variety of examples may help students in their mental constructions.

It is a challenge in undergraduate mathematics education to break students' habits regarding the execution of algorithms and redirect their attention to the structures of representation. This challenge is especially crucial when working with pre-service teachers, the educators of future populations of students. How can we teach students to see what is visible, to notice what is transparent? There are no simple solutions, though some simple 'trivial' questions like the above could be a starting point. But most importantly, if we want students to see what we see, we could start by simply asking them to look, and then to look again. 


\section{Acknowledgments}

For this paper I draw on the collaborative work with two colleagues, Natasa Sirotic and Peter Liljedahl.

\section{References}

[1] Tall, D. and Vinner, S., 1981, Concept image and concept definition in mathematics with particular reference to limts and continuity. Educational Studies in Mathematics, 12, 151.

[2] Zazkis, R. and Liljedahl, P., 2004, Understanding primes: The role of representation. Journal for Research in Mathematics Education, 35(3), 164.

[3] Lesh, R., Behr, M. and Post, M., 1987, Rational number relations and proportions. In: C. Janvier (Ed.) Problems of Representation in the Teaching and Learning of Mathematics (Hillsdale, NJ: Lawrence Erlbaum Associates), pp. 41-58.

[4] Zazkis, R. and Gadowsky, K., 2001, Attending to transparent features of opaque representations of natural numbers. In: A. Cuoco (Ed.) The Roles of Representation in School Mathematics (Reston, VA: NCTM), pp. 146-165.

[5] Zazkis, R. and Sirotic, N., 2004, Making sense of irrational numbers: Focusing on representation. Proceedings of 28th International Conference for Psychology of Mathematics Education, Vol. 4, Bergen, Norway, pp. 497-505.

[6] Cuoco, A. (Ed.), 2001, The Roles of Representation in School Mathematics (Reston, VA: NCTM).

[7] Goldin, G. and Janvier, C. (Eds), 1998, Representations and the psychology of mathematics education, Parts I and II. Special Issue, Journal of Mathematical Behavior, 17(1-2).

[8] Skemp, R., 1986, The Psychology of Learning Mathematics, 2nd Edn (New York: Penguin).

[9] Sirotic, N. and Zazkis, R., 2004, Irrational numbers: Dimensions of knowledge. Proceedings of the North American Chapter Conference for Psychology of Mathematics Education, Toronto, pp. 171-177.

[10] Lamon, S.J., 2001, Presenting and representing: From fractions to rational numbers. In: A. Cuoco (Ed.) The Roles of Representation in School Mathematics (Reston, VA: NCTM), pp. 41-52.

[11] Friedlander, A. and Tabach, M., 2001, Promoting multiple representations in algebra. In: A. Cuoco (Ed.) The Roles of Representation in School Mathematics (Reston, VA: NCTM), pp. 173-184.

[12] Janvier, C., 1987, Representation and understanding: The notion of function as an example. In: C. Janvier (Ed.) Problems of Representation in the Teaching and Learning of Mathematics (Hillsdale, NJ: Lawrence Erlbaum Associates), pp. 67-72.

[13] Even, R., 1998, Factors involved in linking representation of functions. Journal of Mathematical Behavior, 17(1), 105.

[14] Kaput, J., 1991, Notations and representations as mediators of constructive processes. In: E. von Glasersfeld (Ed.) Radical Constructivism in Mathematics Education (Dordrecht: Kluwer Academic), pp. 53-74.

[15] Dubinsky, E., 1991, Reflective abstraction in advanced mathematical thinking. In: D. Tall (Ed.) Advanced Mathematical Thinking (Boston: Kluwer), pp. 95-123.

[16] Sfard, A., 1991, On the dual nature of mathematical conceptions: reflections on processes and objects as different sides of the same coin. Educational Studies in Mathematics., 22, 136.

[17] Rissland, E.L., 1991, Example-based reasoning. In: J.F. Voss, D.N. Parkins and J.W. Segal (Eds) Informal Reasoning in Education (Lawrence Erlbaum Associates), pp. 187208.

[18] Mason, J. and Pimm, D., 1984, Generic examples: Seeing the general in the particular. Educational Studies in Mathematics, 15, 277.

[19] Lakoff, G., 1987, Women, Fire and Dangerous Things (Chicago: University of Chicago Press). 
[20] Mason, J., Burton, L. and Stacey, K., 1982, Thinking Mathematically (Wokingham, UK: Addison-Wesley).

[21] Pólya, G., 1973, How to Solve it? 2nd Edn (Princeton, NJ: Princeton University Press).

[22] Hazzan, O., 1999, Reducing abstraction level when learning abstract algebra. Educational Studies in Mathematics, 40, 71.

[23] Zazkis, R. and Campbell, S., 1996, Prime decomposition: Understanding uniqueness. Journal of Mathematical Behavior, 15, 207.

[24] Sirotic, N., 2004, An inquiry into prospective teachers' understanding of irrational numbers. Unpublished M.Sc. Thesis, Simon Fraser University, Canada.

[25] Zazkis, R., 1998, Odds and ends of odds and evens: An inquiry into students' understanding of even and odd numbers. Educational Studies in Mathematics, 36, 73.

[26] Watson, A. and Mason, J., in press, Learners Generating Examples (Lawrence: Erlbaum). 